\title{
A Note on the Birkhoff Ergodic Theorem
}

\author{
Nikola Sandrić \\ Department of Mathematics \\ Faculty of Civil Engineering, University of Zagreb, 10000 Zagreb, Croatia \\ Email: nsandric@grad.hr
}

April 13, 2017

\begin{abstract}
The classical Birkhoff ergodic theorem states that for an ergodic Markov process the limiting behaviour of the time average of a function (having finite $p$-th moment, $p \geq 1$, with respect to the invariant measure) along the trajectories of the process, starting from the invariant measure, is a.s. and in the $p$-th mean constant and equals to the space average of the function with respect to the invariant measure. The crucial assumption here is that the process starts from the invariant measure, which is not always the case. In this paper, under the assumptions that the underlying process is a Markov process on Polish space, that it admits an invariant probability measure and that its marginal distributions converge to the invariant measure in the $L^{1}$-Wasserstein metric, we show that the assertion of the Birkhoff ergodic theorem holds in the $p$-th mean, $p \geq 1$, for any bounded Lipschitz function and any initial distribution of the process.
\end{abstract}

AMS 2010 Mathematics Subject Classification: 60J05, 60J25

Keywords and phrases: Birkhoff ergodic theorem, ergodicity, Markov process, Wasserstein metric

\section{Introduction}

One of the classical directions in the analysis of Markov processes are limit theorems for Markov processes, such as the law of large numbers, central limit theorem and law of the iterated logarithm. In this paper, we discuss a version of the Birkhoff ergodic theorem (law of large numbers) for a class of Markov processes. Let $\mathbf{M}=\left(\Omega, \mathcal{F},\left\{\mathbb{P}^{x}\right\}_{x \in S},\left\{\mathcal{F}_{t}\right\}_{t \in \mathbb{T}},\left\{\theta_{t}\right\}_{t \in \mathbb{T}},\left\{M_{t}\right\}_{t \in \mathbb{T}}\right.$ ) be a (temporally homogeneous) normal Markov process with state space $(S, \mathcal{S})$, in the sense of [4]. Here, $S$ is a non-empty set, $\mathcal{S}$ is a $\sigma$-algebra of subsets of $S,\left(\Omega, \mathcal{F}, \mathbb{P}^{x}\right)_{x \in S}$ is a family of probability spaces, $\mathbb{T}$ is the time set $\{0,1,2, \ldots\}$ or $[0, \infty),\left\{\mathcal{F}_{t}\right\}_{t \in \mathbb{T}}$ is a filtration on $(\Omega, \mathcal{F})$ (non-decreasing family of sub- $\sigma$-algebras of $\mathcal{F}$ ) and $\left\{\theta_{t}\right\}_{t \in \mathbb{T}}$ is a family of shift operators on $\Omega$ satisfying $M_{t} \circ \theta_{s}=M_{t+s}$ for all $s, t \in \mathbb{T}$. In the case when $\mathbb{T}=[0, \infty)$, assume that $\mathbf{M}$ is progressively measurable (with respect to $\left.\left\{\mathcal{F}_{t}\right\}_{t>0}\right)$, that is, the map $(s, \omega) \longmapsto M_{s}(\omega)$ from $[0, t] \times \Omega$ to $S$ is $\mathcal{B}([0, t]) \times \mathcal{F}_{t} / \mathcal{S}$ measurable for all $t \geq 0$ (measurable in the pair of $\sigma$-algebras $\mathcal{B}([0, t]) \times \mathcal{F}_{t}$ and $\mathcal{S}$, where $\mathcal{B}([0, t])$ denotes the Borel $\sigma$-algebra of subsets of $[0, t])$. Recall that if $S$ is a metric space and $\mathcal{S}$ is the Borel $\sigma$-algebra of subsets of $S$, then $\mathbf{M}$ is progressively measurable provided $t \longmapsto M_{t}(\omega)$ is right continuous for all $\omega \in \Omega$ (see [4, Exercise I.6.13]). Further, denote by $p^{t}(x, d y):=\mathbb{P}^{x}\left(M_{t} \in d y\right), t \in \mathbb{T}, x \in S$, the transition function of $\mathbf{M}$. A measure $\pi(d y)$ on $\mathcal{S}$ is said to be invariant for $\mathbf{M}$ if

$$
\int_{S} p^{t}(x, d y) \pi(d x)=\pi(d y), \quad t \in \mathbb{T} .
$$


A set $B \in \mathcal{F}$ is said to be shift-invariant (for $\mathbf{M}$ ) if $\theta_{t}^{-1} B=B$ for all $t \in \mathbb{T}$. The shift-invariant $\sigma$-algebra $\mathcal{I}$ is a collection of all such shift-invariant sets. Now, the celebrated Birkhoff ergodic theorem asserts that if a Markov process $\mathbf{M}$ admits an invariant (equilibrium) probability measure $\pi(d y)$, then the limiting behaviour of the time average of a function $f \in L^{p}(S, \pi), p \geq 1$, along the trajectories of $\mathbf{M}$, starting from $\pi(d y)$, exists $\mathbb{P}^{\pi}$-a.s. and in $L^{p}\left(\Omega, \mathbb{P}^{\pi}\right)$, it is invariant (that is, it is measurable with respect to $\mathcal{I}$ ) and it is related to the space average of the function with respect to $\pi(d y)$. Here, $L^{p}(S, \pi)\left(L^{p}\left(\Omega, \mathbb{P}^{\pi}\right)\right)$ denotes the space of all measurable functions $f: S \longrightarrow \mathbb{R}$ $(f: \Omega \longrightarrow \mathbb{R})$ with finite $p$-th moment with respect to $\pi(d y)\left(\mathbb{P}^{\pi}(d \omega)\right)$. For a probability measure $\mu(d y)$ on $\mathcal{S}, \mathbb{P}^{\mu}(d \omega)$ is defined as $\mathbb{P}^{\mu}(d \omega):=\int_{S} \mathbb{P}^{y}(d \omega) \mu(d y)$. Also, the collection of all probability measures on $\mathcal{S}$ is denoted by $\mathcal{P}(S)$.

Theorem 1.1. ([12, Theorems 9.6 and 9.8]). Let $\boldsymbol{M}$ be a Markov process with invariant probability measure $\pi(d y)$. Then, for any $f \in L^{p}(S, \pi), p \geq 1$, the following limit holds

$$
\lim _{t \rightarrow \infty} \frac{1}{t} \int_{[0, t)} f\left(M_{s}\right) \tau(d s)=\mathbb{E}^{\pi}\left[f\left(M_{0}\right) \mid \mathcal{I}\right] \quad \mathbb{P}^{\pi} \text {-a.s. and in } L^{p}\left(\Omega, \mathbb{P}^{\pi}\right),
$$

where $\tau(d t)$ is the counting measure when $\mathbb{T}=\{0,1,2, \ldots\}$ and the Lebesgue measure when $\mathbb{T}=$ $[0, \infty)$.

A Markov process $\mathbf{M}$ is said to be ergodic if it possesses an invariant probability measure $\pi(d y)$ and if $\mathcal{I}$ is trivial with respect to $\mathbb{P}^{\pi}(d \omega)$, that is, $\mathbb{P}^{\pi}(B)=0$ or 1 for every $B \in \mathcal{I}$. Now, in addition to the assumptions of Theorem 1.1, if $\mathbf{M}$ is ergodic, then the relation in (1.1) reads as follows

$$
\lim _{t \rightarrow \infty} \frac{1}{t} \int_{[0, t)} f\left(M_{s}\right) \tau(d s)=\int_{S} f(y) \pi(d y) \quad \mathbb{P}^{\pi} \text {-a.s. and in } L^{p}\left(\Omega, \mathbb{P}^{\pi}\right) .
$$

The main assumption in Theorem 1.1 is that the process starts from its equilibrium, which is not always the case. For $f \in L^{p}(S, \pi), p \geq 1$, define

$$
B_{f}:=\left\{\omega \in \Omega: \lim _{t \rightarrow \infty} \frac{1}{t} \int_{[0, t)} f\left(M_{s}(\omega)\right) \tau(d s)=\int_{S} f(y) \pi(d y)\right\} .
$$

Clearly, $B_{f} \in \mathcal{I}$. Thus, in order to conclude that (1.2) holds $\mathbb{P}^{\mu}$-a.s. for any $\mu \in \mathcal{P}(S)$ one expects that $\mathcal{I}$ should be trivial with respect to $\mathbb{P}^{\mu}(d \omega)$ for every $\mu \in \mathcal{P}(S)$. Indeed, in [14, Theorem 17.1.7] it has been proved that the following are equivalent:

(a) $\mathbb{P}^{\mu}\left(B_{f}\right)=1$ for any $f \in L^{p}(S, \pi(d y))$ and $\mu \in \mathcal{P}(S)$;

(b) the shift-invariant $\sigma$-algebra $\mathcal{I}$ is $\mathbb{P}^{\mu}$-trivial for every $\mu \in \mathcal{P}(S)$.

Further, note that in order to conclude (a), it is necessary that $\pi(d y)$ is a unique invariant probability measure for $\mathbf{M}$ (hence, according to [9, Corollary 5.12] or [2, Proposition 2.5], $\mathbf{M}$ is an ergodic Markov process). Namely, if $\mathbf{M}$ admits more than one invariant probability measure, it is ergodic with respect to at least two mutually singular invariant probability measures (see [9, Theorem 5.7]). This leads to the conclusion that, in order to conclude (a), certain additional structural properties of $\mathbf{M}$ will be necessary. In [14, Theorem 17.1.7] it has been also proved that (a) and (b) are equivalent to

(c) $\mathbf{M}$ is a positive Harris recurrent Markov process. 
Recall, a Markov process $\mathbf{M}$ is called $\varphi$-irreducible if for the $\sigma$-finite measure $\varphi(d y)$ on $\mathcal{S}, \varphi(B)>0$ implies

$$
\int_{\mathbb{T}} p^{t}(x, B) \tau(d t)>0, \quad x \in S .
$$

The process $\mathbf{M}$ is called Harris recurrent if it is $\varphi$-irreducible, and $\varphi(B)>0$ implies

$$
\int_{\mathbb{T}} 1_{\left\{M_{t} \in B\right\}} \tau(d t)=\infty \quad \mathbb{P}^{x} \text {-a.s. }
$$

for all $x \in S$. Further, according to [18, Theorem 2.6] every Harris recurrent Markov process admits a unique (up to constant multiplies) invariant (not necessary probability) measure. If the invariant measure is finite, then the process is called positive Harris recurrent; otherwise it is called null Harris recurrent. In the case when the process $\mathbf{M}$ is aperiodic, in [14, Theorem 13.0.1] and [13, Theorem 6.1] it has been proved that (a), (b) and (c) imply

(d) $\mathbf{M}$ is strongly ergodic.

Recall, a discrete-time Markov process $\mathbf{M}$ is called $d$-periodic if $d \geq 1$ is the largest integer for which there is a partition $P_{1}, \ldots, P_{d} \in \mathcal{S}$ of $S$, such that $p\left(x, P_{i+1}\right)=1$ for all $x \in P_{i}$ and all $1 \leq i \leq d-1$, and $p\left(x, P_{1}\right)=1$ for all $x \in P_{d}$. If $d=1$, then $\mathbf{M}$ is called aperiodic. A continuous-time Markov process $\mathbf{M}$ is called aperiodic if it admits an irreducible skeleton chain, that is, there is $\delta>0$ such that the discrete-time Markov process $\left\{M_{\delta n}\right\}_{n \geq 0}$ is irreducible. Further, denote by $B_{b}(S)$ the space of all $\mathbb{R}$-valued, bounded and $\mathcal{S} / \mathcal{B}(\mathbb{R})$ measurable functions on $S$, where $\mathcal{B}(\mathbb{R})$ denotes the Borel $\sigma$-algebra of subsets of $\mathbb{R}$. Also, denote by $d_{T V}$ the total variation metric on $\mathcal{P}(S)$, given by

$$
d_{T V}(\mu(d y), \nu(d y)):=\frac{1}{2} \sup _{f \in B_{b}(S),|f|_{\infty} \leq 1}\left|\int_{S} f(y) \mu(d y)-\int_{S} f(y) \nu(d y)\right|, \quad \mu, \nu \in \mathcal{P}(S),
$$

where $|\cdot|_{\infty}:=\sup _{x \in S}|\cdot|$ denotes the supremum norm on $B_{b}(S)$. Now, a Markov process $\mathbf{M}$ is said to be strongly ergodic if there exists $\pi \in \mathcal{P}(S)$ such that

$$
\lim _{t \rightarrow \infty} d_{T V}\left(p^{t}(x, d y), \pi(d y)\right)=0, \quad x \in S .
$$

Note that the relation in (1.3) automatically implies: (i) $\pi(d y)$ is the only measure satisfying (1.3), (ii) $\pi(d y)$ is necessarily an invariant measure for $\mathbf{M}$ and (iii) $\pi(d y)$ is a unique invariant measure for M. In particular, $\mathbf{M}$ is ergodic. In other words, strong ergodicity implies ergodicity. As one could expect, ergodcity does not in general imply strong ergodicity (see Section 3). Moreover, under aperiodicity assumption, (d) is actually equivalent to (a), (b) and (c). Indeed, assume that $\mathbf{M}$ is strongly ergodic with invariant probability measure $\pi(d y)$. Then, by following [2, Proposition 2.5], for any $x \in S$ and $f \in L^{p}(S, \pi)$ we have that

$$
\begin{aligned}
\left|\mathbb{P}^{x}\left(B_{f}\right)-1\right| & =\left|\mathbb{E}^{x}\left[\mathbb{P}^{M_{t}}\left(B_{f}\right)\right]-\mathbb{E}^{\pi}\left[\mathbb{P}^{M_{t}}\left(B_{f}\right)\right]\right| \\
& =\left|\int_{S} \mathbb{P}^{y}\left(B_{f}\right)\left(p^{t}(x, d y)-\pi(d y)\right)\right| \\
& \leq d_{T V}\left(p^{t}(x, d y), \pi(d y)\right),
\end{aligned}
$$

which entails (a).

However, in many situations the processes we deal with do not meet the properties from (a), (b), (c) and (d). For example, they even do not have to be irreducible (see Section 3 for examples of such processes). Furthermore, in the discrete-time case, in [11] it has been shown that if a Markov process $\mathbf{M}$ has a unique invariant probability measure $\pi(d y)$, then either 
(i) the relation in (1.3) holds $\pi$-a.e., or

(ii) for $\pi$-a.e. $x \in S, \pi(d y)$ is singular with respect to $\sum_{t=1}^{\infty} p^{t}(x, d y)$ and $p^{t}(x, d y)$ converges weakly to $\pi(d y)$.

Clearly, in the later case, $p^{t}(x, d y)$ cannot converge in the total variation metric to $\pi(d y)$. This suggests that the a.s. convergence in (a) is a too strong property (recall that under the aperiodicity assumption (a) and (d) are equivalent). Also, the situation in (ii) suggests that weak convergence might be the key property to be analysed. Accordingly, our main aim is to relax the notion of strong ergodicity and, under these new assumptions, conclude a relation of the form in (1.2) which holds for all $\mu \in \mathcal{P}(S)$.

In the sequel, assume the following additional structural properties of the state space $(S, \mathcal{S})$. There exists a metric $d$ on $S$ inducing $\mathcal{S}$ (hence, $\mathcal{S}$ is the Borel $\sigma$-algebra induced by $d$ ) such that $(S, d)$ is a Polish space, that is, a complete and separable topological space. Further, denote by $\operatorname{Lip}(S)$ the space of all $\mathbb{R}$-valued Lipschitz continuous functions on $S$, that is, functions $f: S \longrightarrow \mathbb{R}$ for which there exists $L_{f} \geq 0$ such that

$$
|f(x)-f(y)| \leq L_{f} d(x, y), \quad x, y \in S .
$$

The best admissible constant $L_{f}$ is then denoted by $|f|_{\text {Lip }}$. Also, denote by $C(S)$ the space of all $\mathbb{R}$-valued continuous functions on $S$ and let $C_{b}(S):=C(S) \cap B_{b}(S)$ be the space of all $\mathbb{R}$ valued continuous and bounded functions on $S$. In this paper we will be concerned with bounded Lipschitz continuous functions only. The reason for that is explained below. Observe that (i) $f \in \operatorname{Lip}(S) \cap B_{b}(S)$ if, and only if, $f(x)$ is Lipschitz continuous with respect to $\bar{d}:=d /(1+d)$ (or $1 \wedge d$ ) and (ii) $\bar{d}$ induces the same topology as $d$. Here, $a \wedge b$ denotes the minimum of $a, b \in \mathbb{R}$. Hence, without loss of generality we may assume that $d$ is bounded, say by 1 , that is, $\sup _{x, y \in S} d(x, y) \leq 1$. In particular, we have $\operatorname{Lip}(S) \subseteq C_{b}(S)$. Now, recall that the Wasserstein metric of order one (also known as the Kantorovich-Rubinshtein metric), denoted by $d_{W}$, is a metric on $\mathcal{P}(S)$ defined by

$$
d_{W}(\mu(d y), \nu(d y)):=\sup _{f \in L i p(S),|f|_{L i p} \leq 1}\left|\int_{S} f(y) \mu(d y)-\int_{S} f(y) \nu(d y)\right|, \quad \mu, \nu \in \mathcal{P}(S) .
$$

Observe that, since $d$ is bounded by $1, d_{W} \leq d_{T V}$ (see [19, Theorem 6.15]). In particular, $d_{W} \leq 1$. Also, if $d$ is the discrete metric on $S, d_{W}=d_{T V}$. Thus, the topology induced by $d_{W}$ is, in general, finer than the topology induced by $d_{T V}$. Recall also that $d_{W}$ is equivalent to the so-called modified Kantorovich-Rubinstein metric and the Lévy-Prohorov metric (see [5, Theorem 8.10.43]). A very important property of the Wasserstein metric is that it metrizes the weak convergence of probability measures, which is due to the fact that the underlying metric is bounded. More precisely, a sequence $\left\{\mu_{n}(d y)\right\}_{n \in \mathbb{N}} \subseteq \mathcal{P}(S)$ converges to $\mu \in \mathcal{P}(S)$ in the Wasserstein topology if, and only if, $\left\{\mu_{n}(d y)\right\}_{n \in \mathbb{N}}$ converges to $\mu(d y)$ weakly, that is,

$$
\lim _{n \rightarrow \infty} \int_{S} f(y) \mu_{n}(d y)=\int_{S} f(y) \mu(d y), \quad f \in C_{b}(S),
$$

(see [19, Corollary 6.13]). For more on the Wasserstein metric we refer the readers to [5] and [19]. We are now in position to state the main results of this paper.

Theorem 1.2. Let $\boldsymbol{M}$ be a Markov process with state space $(S, \mathcal{S})$. Assume that there is $\pi \in \mathcal{P}(S)$ satisfying

$$
\lim _{t \rightarrow \infty} \sup _{s \in \mathbb{T}} \int_{S} d_{W}\left(p^{t}(y, d z), \pi(d z)\right) p^{s}(x, d y)=0, \quad x \in S
$$


Then, for any $p \geq 1, f \in \operatorname{Lip}(S)$ and $\mu \in \mathcal{P}(S)$,

$$
\frac{1}{t} \int_{[0, t)} f\left(M_{s}\right) \tau(d s) \stackrel{L^{p}\left(\Omega, \mathbb{P}^{\mu}\right)}{t \nearrow \infty} \int_{S} f(y) \pi(d y),
$$

where $\underset{t \nearrow \infty}{\stackrel{L^{p}\left(\Omega, \mathbb{P}^{\mu}\right)}{\longrightarrow}}$ denotes the convergence in $L^{p}\left(\Omega, \mathbb{P}^{\mu}\right)$.

Let us remark that

(i) the function $x \longmapsto d_{W}\left(p^{t}(x, d y), \pi(d y)\right)$ is $\mathcal{S} / \mathcal{B}(\mathbb{R})$ measurable for all $t \in \mathbb{T}$ (see the proof of [6, Lemma 4.5] or [10, Theorem 4.8]), hence the relation in (1.5) is well defined.

(ii) the relation in (1.5) is always bounded by 1 .

(iii) for any $t \in \mathbb{T}$ and $x \in S$,

$$
d_{W}\left(p^{t}(x, d y), \pi(d y)\right) \leq \sup _{s \in \mathbb{T}} \int_{S} d_{W}\left(p^{t}(y, d z), \pi(d z)\right) p^{s}(x, d y),
$$

thus (1.5) implies

$$
\lim _{t \longrightarrow \infty} d_{W}\left(p^{t}(x, d y), \pi(d y)\right)=0, \quad x \in S .
$$

(iv) (1.5) trivially holds true if

$$
\lim _{t \longrightarrow \infty} \sup _{x \in S} d_{W}\left(p^{t}(x, d y), \pi(d y)\right)=0
$$

(see [6, Theorems 2.1 and 2.4] and [7, Theorem 5.22] for sufficient conditions ensuring the above relation).

(v) we do not require that $\mathbf{M}$ is irreducible (see Example 3.1).

As a direct consequence of [12, Lemma 3.2 and Proposition 3.12] we also conclude the following.

Corollary 1.3. Let $\boldsymbol{M}$ be a Markov process with state space $(S, \mathcal{S})$. Assume that $\boldsymbol{M}$ satisfies the assumptions from Theorem 1.2. Then,

(i) for any $f \in \operatorname{Lip}(S)$ and $\mu \in \mathcal{P}(S)$, the convergence in (1.6) holds also in probability with respect to $\mathbb{P}^{\mu}(d \omega)$.

(ii) for any $f \in \operatorname{Lip}(S), \mu \in \mathcal{P}(S)$ and sequence $\left\{t_{n}\right\}_{n \in \mathbb{N}} \subseteq \mathbb{T}, t_{n} \nearrow \infty$, there is a further subsequence $\left\{t_{n_{k}}\right\}_{k \in \mathbb{N}} \subseteq\left\{t_{n}\right\}_{n \in \mathbb{N}}$ (possibly depending on $f(x)$ and $\mu(d y)$ ) such that the convergence in (1.6) holds also $\mathbb{P}^{\mu}$-a.s.

(iii) $p^{t}(x, d y), t \in \mathbb{T}$, is weak * mean ergodic, that is,

$$
\frac{1}{t} \int_{[0, t)} \int_{S} p^{t}(x, d y) \mu(d x)
$$

converges weakly to $\pi(d y)$ as $t \nearrow \infty$ for every $\mu \in \mathcal{P}(S)$. 
Further, denote by $C_{c}(S), C_{\infty}(S)$ and $C_{b, u}(S)$ the spaces of all $\mathbb{R}$-valued continuous functions with compact support, vanishing at infinity and uniformly continuous bounded functions on $S$, respectively. Then, according to the Stone-Weierstrass theorem (which implies that $\operatorname{Lip}(S) \cap C_{c}(S)$ is dense in $C_{c}(S)$ with respect to $\left.|\cdot|_{\infty}\right)$ and [15, Theorem 1 and Proposition 6] (which state that $\operatorname{Lip}(S)$ is dense in $C_{b, u}(S)$ with respect $\left.|\cdot|_{\infty}\right)$ we have the following.

Corollary 1.4. Let $\boldsymbol{M}$ be a Markov process with state space $(S, \mathcal{S})$. Assume that $\boldsymbol{M}$ satisfies the assumptions from Theorem 1.2. Then,

(i) the relation in (1.6) holds for all $f \in C_{c}(S)$ and $\mu \in \mathcal{P}(S)$. In particular, if $S$ is compact, then the relation in (1.6) holds for all $f \in C(S)$ and $\mu \in \mathcal{P}(S)$.

(ii) provided $(S, d)$ is also a locally compact space, the relation in (1.6) holds for all $f \in C_{\infty}(S)$ and $\mu \in \mathcal{P}(S)$.

(iii) provided $S$ is also a Hilbert space, the relation in (1.6) holds for all $f \in C_{b, u}(S)$ and $\mu \in \mathcal{P}(S)$.

Directly from the above corollary we conclude the ergodic $L^{p}$-version of the Birkhoff ergodic theorem (relation in (1.2)).

Corollary 1.5. Let $M$ be a Markov process with state space $(S, \mathcal{S})$. In addition to the assumptions from Theorem 1.2, assume that $\pi(d y)$ is an invariant measure for $\boldsymbol{M}$ and that $(S, d)$ is also a locally compact space. Then,

$$
\frac{1}{t} \int_{[0, t)} f\left(M_{s}\right) \tau(d s) \stackrel{L^{p}\left(\Omega, \mathbb{P}^{\pi}\right)}{t \nearrow \infty} \int_{S} f(y) \pi(d y)
$$

holds for any $p \geq 1$ and $f \in L^{p}(S, \pi)$.

Observe that the measure $\pi(d y)$ in the previous corollary is a unique invariant measure for $\mathbf{M}$. Thus, $\mathbf{M}$ is necessarily ergodic. Further, let us remark here that if $\mathbf{M}$ is a strong Feller process, that is, if $x \longmapsto \int_{S} f(y) p^{t}(x, d y)$ is a $C_{b}(S)$ function for every $t \in \mathbb{T} \backslash\{0\}$ and $f \in B_{b}(S)$, then, under (1.7), (a) is easily concluded from the second line in (1.4) and the fact that the Wasserstein metric metrizes the weak convergence of probability measures. In particular, as we have already commented, (under aperiodicity assumption) this implies strong ergodicity of $\mathbf{M}$. For sufficient conditions which ensure the strong Feller property of Markov processes see [17]. Further, note that, similarly as in the case of the total variation metric, the condition in (1.7), together with [3, Theorem 1.2], implies that $\pi(d y)$ is the only measure satisfying (1.7) (and (1.5)). On the other hand, it is not completely clear that (1.7) (or (1.5)) automatically ensures invariance (with respect to $\mathbf{M})$ of $\pi(d y)$. However, if, in addition, we assume that $\mathbf{M}$ is a $C_{b}$-Feller process, that is, if $x \longmapsto \int_{S} f(y) p^{t}(x, d y)$ is a $C_{b}(S)$ function for every $t \in \mathbb{T}$ and $f \in C_{b}(S)$, then (1.7) implies invariance of $\pi(d y)$. Indeed, for any $t \in \mathbb{T}, x \in S$ and $f \in C_{b}(S)$, we have that

$$
\begin{aligned}
\int_{S} f(y) \pi(d y) & =\lim _{s \rightarrow \infty} \int_{S} f(y) p^{s+t}(x, d y) \\
& =\lim _{s \rightarrow \infty} \int_{S} \int_{S} f(y) p^{t}(z, d y) p^{s}(x, d z) \\
& =\int_{S} f(y) \int_{S} p^{t}(z, d y) \pi(d z) .
\end{aligned}
$$

The assertion now follows from [3, Theorem 1.2]. For conditions ensuring that a Markov process is a $C_{b}$-Feller process see [16]. Another condition ensuring that (1.7) (or (1.5)) implies invariance of $\pi(d y)$ is contractivity of $p^{t}(x, d y)$ with respect to $d_{W}$, that is,

$$
d_{W}\left(p^{t}\left(x_{1}, d y\right), p^{t}\left(x_{2}, d y\right)\right) \leq d\left(x_{1}, x_{2}\right), \quad t \in \mathbb{T}, x_{1}, x_{2} \in S .
$$


To see this, first, according to the proof of [6, Lemma 4.5], the above relation yields that for any $t \in \mathbb{T}$ and $\mu, \nu \in \mathcal{P}(S)$, we have that

$$
d_{W}\left(\int_{S} p^{t}(x, d y) \mu(d x), \int_{S} p^{t}(x, d y) \nu(d x)\right) \leq d_{W}(\mu(d y), \nu(d y)) .
$$

In particular, by employing (1.7), we have that

$$
\lim _{s \rightarrow \infty} d_{W}\left(\int_{S} p^{t}(x, d y) \pi(d x), p^{s+t}(x, d y)\right) \leq \lim _{s \rightarrow \infty} d_{W}\left(\pi(d y), p^{s}(x, d y)\right)=0, \quad t \in \mathbb{T}, x \in S,
$$

which yields,

$$
\int_{S} f(y) \int_{S} p^{t}(x, d y) \pi(d x)=\lim _{s \rightarrow \infty} \int_{S} f(y) p^{s+t}(x, d y)=\int_{S} f(y) \pi(d y), \quad t \in \mathbb{T}, x \in S .
$$

Recall that every Markov semigroup is contractive with respect to $d_{T V}$ (in the sense (1.9)). Finally, if $\pi(d y)$ is an invariant probability measure of $\mathbf{M}$ satisfying (1.7), then, just by applying [3, Theorem 1.2 ], we easily see that $\pi(d y)$ is actually a unique invariant probability measure for $\mathbf{M}$. Consequently, $\mathbf{M}$ is ergodic.

Based on the previous discussions, it is tempting to conclude that (1.5) in Theorem 1.2 might be replaced by (1.7) (as in the strong ergodicity situation). However, it is not completely clear that (1.7) alone is sufficient to conclude the assertion of Theorem 1.2. In the following theorem we prove that, in addition to (1.7), if the transition function $p^{t}(x, d y), t \in \mathbb{T}$, is Lipschitz, that is, $x \longmapsto \int_{S} f(y) p^{t}(x, d y)$ is Lipschitz for every $f \in \operatorname{Lip}(S)$ and $t \in \mathbb{T}$, then the assertion of Theorem 1.2 holds true. For more on Markov processes with Lipschitz transition function we refer the readers to $[1]$.

Theorem 1.6. Let $\boldsymbol{M}$ be a Markov process with state space $(S, \mathcal{S})$. Assume that there is $\pi \in \mathcal{P}(S)$ satisfying (1.7), and for every $f \in \operatorname{Lip}(S)$ and $t \in \mathbb{T}$, the function

$$
F_{f, t}(x):=\int_{S} f(y) p^{t}(x, d y), \quad x \in S,
$$

is also in $\operatorname{Lip}(S)$ with $\left|F_{f, t}\right|_{\text {Lip }} \leq C_{f}$, where the constant $C_{f}$ depends only on $f(x)$. Then, for any $f \in \operatorname{Lip}(S)$ and $\mu \in \mathcal{P}(S), M$ satisfies the relation in (1.6).

As we have already commented, one feature that distinguishes the total variation metric among other metrics (on $\mathcal{P}(S)$ ) is that, for any Markov transition function, one always has the contraction property in (1.9). On the other hand, the Wasserstein metric may not be contracting in general. It is therefore natural to focus only on Wasserstein metrics that are contracting for $\mathbf{M}$ (in the sense of relation (1.8)). Also, let us remark that it has been observed in [10] that (1.8) is essential (but not sufficient) in obtaining the convergence to an unique invariant probability measure (see [10] for detailed discussion on this property). As a direct consequence of the contraction property in (1.8) we get that

(i) the function $t \longmapsto d_{W}\left(p^{t}(x, d y), \pi(d y)\right)$ is non-increasing for all $x \in S$.

(ii) for every $f \in \operatorname{Lip}(S)$ and $t \in \mathbb{T}, F_{f, t} \in \operatorname{Lip}(S)$ and $\left|F_{f, t}\right|_{\text {Lip }} \leq|f|_{\text {Lip }}$.

Thus, as a direct consequence of Theorem 1.6 we get the following.

Theorem 1.7. Let $\boldsymbol{M}$ be a Markov process with state space $(S, \mathcal{S})$. Assume that there is $\pi \in \mathcal{P}(S)$ satisfying (1.7) and (1.8) (hence, as we have already commented, $\pi(d y)$ is necessarily a unique invariant measure for $\boldsymbol{M})$. Then, for any $f \in \operatorname{Lip}(S)$ and $\mu \in \mathcal{P}(S), \boldsymbol{M}$ satisfies the relation in (1.6). 


\section{Proof of Theorems 1.2 and 1.6 and Corollary 1.5}

In this section, we prove Theorems 1.2 and 1.6 and Corollary 1.5. Before the proofs, we introduce some notation that will be used in the sequel. For $\mu \in \mathcal{P}(S)$ and $f \in B_{b}(S)$, we write $\mu(f)$ for $\int_{S} f(y) \mu(d y)$. Also, with $\left\{P_{t}\right\}_{t \in \mathbb{T}}$ is denoted the semigroup of $\mathbf{M}$ on $B_{b}(S)$, that is, $P_{t} f(x):=$ $\int_{S} f(y) p^{t}(x, d y), t \in \mathbb{T}, x \in S, f \in B_{b}(S)$.

Proof of Theorem 1.2. First, observe that it suffices to prove the assertion only for the Dirac measures as the initial distributions of $\mathbf{M}$. Also, since for each $p \geq 1$ and $f \in \operatorname{Lip}(S)$ (recall that $\left.\operatorname{Lip}(S) \subseteq C_{b}(S)\right)$ the family

$$
\left\{\left|\frac{1}{t} \int_{[0, t)} f\left(M_{s}\right) \tau(d s)\right|^{p}: t \in \mathbb{T}\right\}
$$

is uniformly integrable, it suffices to consider the case when $p=2$ only (see [12, Proposition 3.12]). For arbitrary $x \in S$ and $f \in \operatorname{Lip}(S)$ we have that

$$
\begin{aligned}
& \mathbb{E}^{x}\left[\left(\frac{1}{t} \int_{[0, t)} f\left(M_{s}\right) \tau(d s)-\pi(f)\right)^{2}\right] \\
& =\mathbb{E}^{x}\left[\left(\frac{1}{t} \int_{[0, t)} f\left(M_{s}\right) \tau(d s)\right)^{2}\right]-\frac{2 \pi(f)}{t} \int_{[0, t)} P_{s} f(x) \tau(d s)+\pi(f)^{2} .
\end{aligned}
$$

Assume first that $\mathbb{T}=[0, \infty)$. Thus,

$$
\mathbb{E}^{x}\left[\left(\frac{1}{t} \int_{[0, t)} f\left(M_{s}\right) \tau(d s)-\pi(f)\right)^{2}\right]=\mathbb{E}^{x}\left[\left(\int_{0}^{1} f\left(M_{s t}\right) d s\right)^{2}\right]-2 \pi(f) \int_{0}^{1} P_{s t} f(x) d s+\pi(f)^{2} .
$$

In the sequel, without loss of generality, assume that $|f|_{\text {Lip }} \leq 1$. Otherwise, divide $f(x)$ by $|f|_{\text {Lip }}$. Next, note that

$$
\lim _{t \rightarrow \infty} \int_{0}^{1} P_{s t} f(x) d s=\pi(f) .
$$

Indeed, by assumption,

$$
\lim _{t \rightarrow \infty} d_{W}\left(p^{t}(x, d y), \pi(d y)\right) \leq \lim _{t \rightarrow \infty} \sup _{s \in \mathbb{T}} P_{s} d_{W}\left(p^{t}(\cdot, d z), \pi(d z)\right)(x)=0
$$

In particular,

$$
\lim _{t \rightarrow \infty}\left|P_{t} f(x)-\pi(f)\right|=0 .
$$

Thus, the claim is a direct consequence of the dominated convergence theorem. Note that the above relation also holds for all $x \in S$ and $f \in C_{b}(S)$ (the Wasserstein metric metrizes the weak convergence of probability measures). Consequently, in order to prove the assertion, it suffices to prove that

$$
\lim _{t \rightarrow \infty} \mathbb{E}^{x}\left[\left(\int_{0}^{1} f\left(M_{s t}\right) d s\right)^{2}\right]=\pi(f)^{2} .
$$


By Fubini's theorem and the Markov property we have that

$$
\begin{aligned}
& \left|\mathbb{E}^{x}\left[\left(\int_{0}^{1} f\left(M_{s t}\right) d s\right)^{2}\right]-\pi(f)^{2}\right| \\
& =\left|2 \mathbb{E}^{x}\left[\int_{0}^{1} \int_{0}^{s} f\left(M_{s t}\right) f\left(M_{u t}\right) d u d s\right]-\pi(f)^{2}\right| \\
& =\left|2 \int_{0}^{1} \int_{0}^{s} P_{u t}\left(f \mathbb{E}\left[f\left(M_{(s-u) t}\right)\right]\right)(x) d u d s-\pi(f)^{2}\right| \\
& \leq 2\left|\int_{0}^{1} \int_{0}^{s}\left(P_{u t}\left(f P_{(s-u) t} f\right)(x)-P_{u t}(f \pi(f))(x)\right) d u d s\right|+2\left|\int_{0}^{1} \int_{0}^{s}\left(P_{u t}(f \pi(f))(x)-\pi(f)^{2}\right) d u d s\right| \\
& =2\left|\int_{0}^{1} \int_{0}^{s} P_{u t}\left(P_{(s-u) t} f-\pi(f)\right) f(x) d u d s\right|+2\left|\pi(f) \int_{0}^{1} \int_{0}^{s}\left(P_{u t} f(x)-\pi(f)\right) d u d s\right| \\
& \leq 2|f|_{\infty} \int_{0}^{1} \int_{0}^{s} P_{u t}\left|P_{(s-u) t} f-\pi(f)\right|(x) d u d s+2|f|_{\infty} \int_{0}^{1} \int_{0}^{s}\left|P_{u t} f(x)-\pi(f)\right| d u d s \\
& \leq 2|f|_{\infty} \int_{0}^{1} \int_{0}^{s} \sup _{v \in[0, \infty)} P_{v} d_{W}\left(p^{(s-u) t}(\cdot, d z), \pi(d z)\right)(x) d u d s+2|f|_{\infty} \int_{0}^{1} \int_{0}^{s}\left|P_{u t} f(x)-\pi(f)\right| d u d s .
\end{aligned}
$$

Now, by letting $t \nearrow \infty$ the assertion follows.

In the discrete-time case we have

$$
\frac{1}{t} \sum_{n=0}^{t-1} f\left(M_{n}\right)=\frac{1}{t} \int_{0}^{t} f\left(M_{[s]}\right) d s=\int_{0}^{1} f\left(M_{[s t]}\right) d s, \quad t \in\{1,2,3, \ldots\}
$$

where $[a]$ denotes the integer part of $a \in \mathbb{R}$. Hence,

$$
\mathbb{E}^{x}\left[\left(\frac{1}{t} \int_{[0, t)} f\left(M_{s}\right) \tau(d s)-\pi(f)\right)^{2}\right]=\mathbb{E}^{x}\left[\left(\int_{0}^{1} f\left(M_{[s t]}\right) d s\right)^{2}\right]-2 \pi(f) \int_{0}^{1} P_{[s t]} f(x) d s+\pi(f)^{2} .
$$

Now, the proof of the assertion proceeds analogously as in the continuous-time case.

Proof of Corollary 1.5. Let $f \in L^{p}(S, \pi), p \geq 1$. Then, by [8, Proposition 7.9], for any $\varepsilon>0$ there is $f_{\varepsilon} \in C_{c}(S)$ such that

$$
\left(\int_{S}\left|f(x)-f_{\varepsilon}(x)\right|^{p} \pi(d x)\right)^{1 / p}<\frac{\varepsilon}{2} .
$$

Further, by Fubini's theorem and Jensen's inequality, we have that

$$
\begin{aligned}
& \left(\mathbb{E}^{\pi}\left[\left|\frac{1}{t} \int_{[0, t)} f\left(M_{s}\right) \tau(d s)-\pi(f)\right|^{p}\right]\right)^{1 / p} \\
& \leq\left(\mathbb{E}^{\pi}\left[\left\lfloor\frac{1}{t} \int_{[0, t)} f\left(M_{s}\right) \tau(d s)-\left.\frac{1}{t} \int_{[0, t)} f_{\varepsilon}\left(M_{s}\right) \tau(d s)\right|^{p}\right]\right)^{1 / p}\right. \\
& +\left(\mathbb{E}^{\pi}\left[\left|\frac{1}{t} \int_{[0, t)} f_{\varepsilon}\left(M_{s}\right) \tau(d s)-\pi\left(f_{\varepsilon}\right)\right|^{p}\right]\right)^{1 / p}+\left(\mathbb{E}^{\pi}\left[\left|\pi\left(f_{\varepsilon}\right)-\pi(f)\right|^{p}\right]\right)^{1 / p}
\end{aligned}
$$




$$
\begin{aligned}
\leq & \left(\frac{1}{t} \int_{[0, t)} \mathbb{E}^{\pi}\left[\left|f\left(M_{s}\right)-f_{\varepsilon}\left(M_{s}\right)\right|^{p}\right] \tau(d s)\right)^{1 / p}+\left(\mathbb{E}^{\pi}\left[\left|\frac{1}{t} \int_{[0, t)} f_{\varepsilon}\left(M_{s}\right) \tau(d s)-\pi\left(f_{\varepsilon}\right)\right|^{p}\right]\right)^{1 / p} \\
& +\left(\int_{S}\left|f_{\varepsilon}(x)-f(x)\right|^{p} \pi(d x)\right)^{1 / p} \\
< & \left(\int_{S}\left|f(x)-f_{\varepsilon}(x)\right|^{p} \pi(d x)\right)^{1 / p}+\left(\mathbb{E}^{\mu}\left[\left|\frac{1}{t} \int_{[0, t)} f_{\varepsilon}\left(M_{s}\right) \tau(d s)-\pi\left(f_{\varepsilon}\right)\right|^{p}\right]\right)^{1 / p}+\frac{\varepsilon}{2} \\
< & +\left(\mathbb{E}^{\mu}\left[\left|\frac{1}{t} \int_{[0, t)} f_{\varepsilon}\left(M_{s}\right) \tau(d s)-\pi\left(f_{\varepsilon}\right)\right|^{p}\right]\right)^{1 / p} .
\end{aligned}
$$

Finally, by employing Corollary 1.4 (i) we conclude

$$
\lim _{t \rightarrow \infty}\left(\mathbb{E}^{\mu}\left[\left|\frac{1}{t} \int_{[0, t)} f\left(M_{s}\right) \tau(d s)-\pi(f)\right|^{p}\right]\right)^{1 / p}<\varepsilon,
$$

which proofs the desired result.

Proof of Theorem 1.6. We proceed similarly as in Theorem 1.2. We discuss only the continuoustime case. Again, the assertion will follow if we prove that

$$
\lim _{t \rightarrow \infty} \mathbb{E}^{x}\left[\left(\int_{0}^{1} f\left(M_{s t}\right) d s\right)^{2}\right]=\pi(f)^{2}, \quad x \in S .
$$

By assumption,

$$
\lim _{t \rightarrow \infty} d_{W}\left(p^{t}(x, d y), \pi(d y)\right)=0 \quad \text { and } \quad \lim _{t \rightarrow \infty} \int_{S} d_{W}\left(p^{t}(y, d z), \pi(d z)\right) \pi(d y)=0 .
$$

Recall that $\sup _{t \in \mathbb{T}, x \in S} d_{W}\left(p^{t}(x, d y), \pi(d y)\right) \leq 1$, hence the later condition is a consequence of the dominated convergence theorem. In particular,

$$
\lim _{t \rightarrow \infty}\left|P_{t} f(x)-\pi(f)\right|=0
$$

Again, similarly as before, by Fubini's theorem and the Markov property, we have

$$
\begin{aligned}
& \left|\mathbb{E}^{x}\left[\left(\int_{0}^{1} f\left(M_{s t}\right) d s\right)^{2}\right]-\pi(f)^{2}\right| \\
& \leq 2\left|\int_{0}^{1} \int_{0}^{s} P_{u t}\left(P_{(s-u) t} f-\pi(f)\right) f(x) d u d s\right|+2\left|\pi(f) \int_{0}^{1} \int_{0}^{s}\left(P_{u t} f(x)-\pi(f)\right) d u d s\right| \\
& \leq 2\left|\int_{0}^{1} \int_{0}^{s} P_{u t}\left(P_{(s-u) t} f-\pi(f)\right) f(x) d u d s\right|+2|f|_{\infty} \int_{0}^{1} \int_{0}^{s}\left|P_{u t} f(x)-\pi(f)\right| d u d s \\
& \leq 2|f|_{\infty} \int_{0}^{1} \int_{0}^{s}\left|P_{u t} f(x)-\pi(f)\right| d u d s+2\left|\int_{0}^{1} \int_{0}^{s}\left(P_{u t}\left(f P_{(s-u) t} f\right)(x)-\pi\left(f P_{(s-u) t} f\right)\right) d u d s\right| \\
& \quad+2\left|\int_{0}^{1} \int_{0}^{s}\left(\pi\left(f P_{(s-u) t} f\right)-\pi(f) P_{u t} f(x)\right) d u d s\right| .
\end{aligned}
$$


Now, since $\operatorname{Lip}(S) \subseteq C_{b}(S), P_{t} f \in \operatorname{Lip}(S)$ (with $\left|P_{t} f\right|_{\text {Lip }} \leq C_{f}$ ) for every $f \in \operatorname{Lip}(S)$ and $t \in \mathbb{T}$, and for $f, g \in \operatorname{Lip}(S), f g \in \operatorname{Lip}(s)$ (with $|f g|_{\text {Lip }} \leq|f|_{\infty}|f|_{\text {Lip }}+|g|_{\infty}|g|_{\text {Lip }}$ ), we conclude that

$$
\lim _{t \rightarrow \infty}\left|\mathbb{E}^{x}\left[\left(\int_{0}^{1} f\left(M_{s t}\right) d s\right)^{2}\right]-\pi(f)^{2}\right| \leq 2 \lim _{t \rightarrow \infty}\left|\int_{0}^{1} \int_{0}^{s}\left(\pi\left(f P_{(s-u) t} f\right)-\pi(f) P_{u t} f(x)\right) d u d s\right| .
$$

Finally, we have

$$
\begin{aligned}
& 2\left|\int_{0}^{1} \int_{0}^{s}\left(\pi\left(f P_{(s-u) t} f\right)-\pi(f) P_{u t} f(x)\right) d u d s\right| \\
& \leq 2\left|\int_{0}^{1} \int_{0}^{s}\left(\pi\left(f P_{(s-u) t} f\right)-\pi(f)^{2}\right) d u d s\right|+2\left|\int_{0}^{1} \int_{0}^{s}\left(\pi(f)^{2}-\pi(f) P_{u t} f(x)\right) d u d s\right| \\
& \leq 2\left|\int_{0}^{1} \int_{0}^{s} \int_{S}\left(P_{(s-u) t} f(y)-\pi(f)\right) f(y) \pi(d y) d u d s\right|+2|f|_{\infty} \int_{0}^{1} \int_{0}^{s}\left|\pi(f)-P_{u t} f(x)\right| d u d s \\
& =2|f|_{\infty} \int_{0}^{1} \int_{0}^{s} \int_{S}\left|P_{(s-u) t} f(y)-\pi(f)\right| \pi(d y) d u d s+2|f|_{\infty} \int_{0}^{1} \int_{0}^{s}\left|\pi(f)-P_{u t} f(x)\right| d u d s,
\end{aligned}
$$

which concludes the proof.

\section{$3 \quad$ Examples}

In this section, we give some applications of Theorems 1.2, 1.6 and 1.7.

Example 3.1. Let $\left\{X_{n}\right\}_{n \geq 1}$ be a sequence of i.i.d. $\mathbb{R}$-valued random variables on a probability space $(\Omega, \mathcal{F}, \mathbb{P})$, satisfying $\mathbb{P}\left(X_{n}=0\right)=\mathbb{P}\left(X_{n}=1 / 2\right)=1 / 2$. Define

$$
M_{n+1}:=\frac{1}{2} M_{n}+X_{n+1}, \quad n \geq 0, M_{0} \in[0,1] .
$$

Clearly, $\mathbf{M}:=\left\{M_{n}\right\}_{n \geq 0}$ is a Markov process with state space $([0,1], \mathcal{B}([0,1]))$ and transition function $p(x, d y):=\mathbb{P}\left(X_{1}+x / 2 \in d y\right), x \in[0,1]$. Also, it is easy to see that the Lebesgue measure (on $\mathcal{B}([0,1]))$ is invariant for $\mathbf{M}$ and $\mathbf{M}$ is ergodic with respect to $\operatorname{Leb}(d y)$. In particular, the relation in (1.2) holds true. However, observe that, since $\operatorname{Leb}(d y)$ is singular with respect to $p(x, d y), \mathbf{M}$ is not strongly ergodic. Let $d(x, y):=|x-y|, x, y \in[0,1]$. Now, by a straightforward computation, we get

$$
d_{W}\left(p\left(x_{1}, d y\right), p\left(x_{2}, d y\right)\right) \leq \frac{d\left(x_{1}, x_{2}\right)}{2}, \quad x_{1}, x_{2} \in[0,1],
$$

which, together with the proof of [6, Lemma 4.5], yields that for all $n \geq 1$ and $x, x_{1}, x_{2} \in[0,1]$,

$$
d_{W}\left(p^{n}\left(x_{1}, d y\right), p^{n}\left(x_{2}, d y\right)\right) \leq \frac{d\left(x_{1}, x_{2}\right)}{2^{n}} \quad \text { and } \quad d_{W}\left(p^{n}(x, d y), \operatorname{Leb}(d y)\right) \leq \frac{1}{2^{n}} .
$$

Thus, Theorem 1.2 (and Theorems 1.6 and 1.7) applies. Also, note that $\mathbf{M}$ is not irreducible.

Example 3.2. ([10, Section 3] and $[6$, Example 3.4]). Let $\mathcal{C}:=C([-1,0], \mathbb{R})$ be the space of all continuous functions from $[-1,0]$ to $\mathbb{R}$ endowed with the supremum norm. For $t \geq 0$ and a function $f(s)$ defined on $[t-1, t]$, we write $f^{t}(s):=f(s+t), s \in[-1,0]$. Consider the following stochastic functional differential equation (the so-called stochastic delay equation)

$$
d M_{t}=-M_{t} d t+G\left(M_{t-1}\right) d B_{t}, \quad t \geq 0, M^{0} \in \mathcal{C},
$$


where $G: \mathbb{R} \longrightarrow \mathbb{R}$ and $\left\{B_{t}\right\}_{t \geq 0}$ is a standard one-dimensional Brownian motion. Now, by assuming that $G(u)$ is bounded, Lipschitz continuous, strictly positive and strictly increasing, in [10, Theorems 3.1 and 4.8] it has been proven that the equation in (3.1) admits a unique strong solution $\mathbf{M}:=\left\{M^{t}\right\}_{t \geq 0}$ which is a strong Markov and $C_{b}$-Feller process with state space $(\mathcal{C}, \mathcal{B}(\mathcal{C}))$. Furthermore, due to [10, Corollary 4.11], $\mathbf{M}$ possesses a unique invariant probability measure $\pi(d y)$ which is singular with respect to the transition function $p^{t}(x, d y)$ of $\mathbf{M}$. Hence, $\mathbf{M}$ is not strongly ergodic. Next, fix $\delta>0$ and define $d(x, y):=1 \wedge|x-y|_{\infty} / \delta, x, y \in \mathcal{C}$. Then, according to [10, Theorem 4.8] (see also [6, Example 3.2]), for suitable (small enough) constant $\delta>0$ there exist a constant $c>0$ and Borel function $C: \mathcal{C} \longrightarrow[0, \infty)$, such that (1.8) holds,

$$
d_{W}\left(p^{t}(x, d y), \pi(d y)\right) \leq C(x) e^{-c t} \quad \text { and } \quad \sup _{s \geq 0} P_{s} C(x)<\infty, \quad t \geq 0, x \in \mathcal{C},
$$

hence we are in the situation of Theorem 1.2 (and Theorems 1.6 and 1.7).

\section{Acknowledgement}

This work has been supported in part by the Croatian Science Foundation under Project 3526 and NEWFELPRO Programme under Project 31. The author thanks the anonymous reviewer for careful reading of the paper and for helpful comments that led to improvement in the presentation.

\section{References}

[1] R. F. Bass. Markov processes with Lipschitz semigroups. Trans. Amer. Math. Soc., 267(1):307320, 1981.

[2] R. N. Bhattacharya. On the functional central limit theorem and the law of the iterated logarithm for Markov processes. Z. Wahrsch. Verw. Gebiete, 60(2):185-201, 1982.

[3] P. Billingsley. Convergence of probability measures. John Wiley \& Sons, Inc., New York, second edition, 1999.

[4] R. M. Blumenthal and R. K. Getoor. Markov processes and potential theory. Academic Press, New York-London, 1968.

[5] V. I. Bogachev. Measure theory. Vol. II. Springer-Verlag, Berlin, 2007.

[6] O. Butkovsky. Subgeometric rates of convergence of Markov processes in the Wasserstein metric. Ann. Appl. Probab., 24(2):526-552, 2014.

[7] M.-F. Chen. From Markov chains to non-equilibrium particle systems. World Scientific Publishing Co., Inc., River Edge, NJ, second edition, 2004.

[8] G. B. Folland. Real analysis. John Wiley \& Sons, Inc., New York, 1984.

[9] M. Hairer. Ergodic properties of Markov processes. Lecture notes, University of Warwick. Available on http://www.hairer.org/notes/Markov.pdf, 2006.

[10] M. Hairer, J. C. Mattingly, and M. Scheutzow. Asymptotic coupling and a general form of Harris' theorem with applications to stochastic delay equations. Probab. Theory Related Fields, 149(1-2):223-259, 2011. 
[11] O. Hernández-Lerma and J. B. Lasserre. On the classification of Markov chains via occupation measures. Appl. Math. (Warsaw), 27(4):489-498, 2000.

[12] O. Kallenberg. Foundations of modern probability. Springer-Verlag, New York, 1997.

[13] S. P. Meyn and R. L. Tweedie. Stability of Markovian processes. II. Continuous-time processes and sampled chains. Adv. in Appl. Probab., 25(3):487-517, 1993.

[14] S. P. Meyn and R. L. Tweedie. Markov chains and stochastic stability. Cambridge University Press, Cambridge, second edition, 2009.

[15] R. Miculescu. Approximations by Lipschitz functions generated by extensions. Real Anal. Exchange, 28(1):33-40, 2002/03.

[16] R. L. Schilling. Conservativeness and extensions of feller semigroups. Positivity, 2:239-256, 1998.

[17] R. L. Schilling and J. Wang. Strong Feller continuity of Feller processes and semigroups. Infin. Dimens. Anal. Quantum Probab. Relat. Top., 15(2):1250010, 28, 2012.

[18] R. L. Tweedie. Topological conditions enabling use of Harris methods in discrete and continuous time. Acta Appl. Math., 34(1-2):175-188, 1994.

[19] C. Villani. Optimal transport. Springer-Verlag, Berlin, 2009. 